PROCEEDINGS OF THE

AMERICAN MATHEMATICAL SOCIETY

Volume 128, Number 1, Pages 157-160

S 0002-9939(99)05011-X

Article electronically published on June 21, 1999

\title{
AN EXISTENCE THEOREM FOR SYSTEMS OF BOUNDARY VALUE PROBLEMS
}

\author{
GERD HERZOG AND ROLAND LEMMERT
}

(Communicated by Hal L. Smith)

\begin{abstract}
We prove an existence theorem for $u^{\prime \prime}(t)=f\left(t, u(t), u^{\prime}(t)\right), u(0)=$ $u_{0}, u(1)=u_{1}$ in $\mathbb{R}^{n}$, using the shooting method. The function $f$ is supposed to be asymptotically linear.
\end{abstract}

\section{INTRODUCTION}

Let $\|\cdot\|$ denote the Euclid norm on $\mathbb{R}^{n}$. By $\||\cdot|\|$ we denote the corresponding maximum norm on $C\left([0,1], \mathbb{R}^{n}\right)$. The set of all real $n \times n$ matrices is denoted by $M^{n \times n}(\mathbb{R})$, and let $\|A\|, A \in M^{n \times n}(\mathbb{R})$ be the matrix norm corresponding to the Euclid norm.

Let $f:[0,1] \times \mathbb{R}^{2 n} \rightarrow \mathbb{R}^{n}$ be a continuous function. We consider the Dirichlet boundary value problem

$$
u^{\prime \prime}(t)=f\left(t, u(t), u^{\prime}(t)\right), \quad u(0)=u_{0}, u(1)=u_{1} .
$$

If $f$ is such that the initial value problem

$$
v^{\prime \prime}(t)=f\left(t, v(t), v^{\prime}(t)\right), \quad v(0)=u_{0}, v^{\prime}(0)=s,
$$

is uniquely solvable on $[0,1]$ for each $s \in \mathbb{R}^{n}$ with solution $v(t, s)$, the function $q: \mathbb{R}^{n} \rightarrow \mathbb{R}^{n}, q(s)=v(1, s)$ is called the shooting function of $u^{\prime \prime}=f\left(t, u, u^{\prime}\right)$ (with respect to $u_{0}$ ), and obviously the solution of (2) is a solution of (1) if and only if $q(s)=u_{1}$.

We will prove the following theorem:

Theorem 1. Let $f:[0,1] \times \mathbb{R}^{2 n} \rightarrow \mathbb{R}^{n}$ be continuous and let $A, B:[0,1] \rightarrow$ $M^{n \times n}(\mathbb{R})$ be continuous matrix functions such that

1. $\lim _{\|x\|+\|p\| \rightarrow \infty} \frac{\|f(\cdot, x, p)-A(\cdot) x-B(\cdot) p\| \mid}{\|x\|+\|p\|}=0$;

2. The boundary value problem $w^{\prime \prime}(t)=A(t) w(t)+B(t) w^{\prime}(t), w(0)=w(1)=0$ has only the trivial solution.

Then problem (1) is solvable for each $u_{0}, u_{1} \in \mathbb{R}^{n}$.

Note that 1 . is more general than it might look at first glance; e.g., if $f$ satisfies 1. and $g$ is bounded, then $f+g$ satisfies 1 . as well.

Received by the editors March 10, 1998.

1991 Mathematics Subject Classification. Primary 34B15.

Key words and phrases. Dirichlet problem, shooting function, existence theorem.

(C)1999 American Mathematical Society 
Theorem 1 is well known for the case $n=1$ and is due to Perow; see [6]. The proof for this case uses typical methods of one and two dimensional analysis, for example, it involves polar coordinates, and cannot be transfered in an obvious way to systems of boundary value problems. To prove Theorem 1 we will approximate problem (1) by boundary value problems with surjective shooting function in a uniform way to obtain a solution of problem (1) itself. For further investigations of existence results with the shooting method see [4], [5].

Proof. To prove Theorem 1 we will use the following propositions.

Proposition 1. Let $A, B:[0,1] \rightarrow M^{n \times n}(\mathbb{R})$ be continuous matrix functions such that the boundary value problem $w^{\prime \prime}(t)=A(t) w(t)+B(t) w^{\prime}(t), w(0)=w(1)=0$, is uniquely solvable (by $w=0)$. Then:

1.) The boundary value problem $w^{\prime \prime}(t)=A(t) w(t)+B(t) w^{\prime}(t)+h(t), w(0)=$ $w(1)=0$ is uniquely solvable for each $h \in C\left([0,1], \mathbb{R}^{n}\right)$, and there is a constant $\alpha \geq 0$ such that

$$
\max \left\{\|w(t)\|,\left\|w^{\prime}(t)\right\|\right\} \leq \alpha\|\mid h\| \|, \quad t \in[0,1] .
$$

2.) The boundary value problem $w^{\prime \prime}(t)=A(t) w(t)+B(t) w^{\prime}(t), w(0)=w_{0}$, $w(1)=w_{1}$ is uniquely solvable for each $w_{0}, w_{1} \in \mathbb{R}^{n}$ and the solution $w$ depends continuously on $w_{0}, w_{1}$.

For a proof of Proposition 1 see for example [3], p. 418ff.

Proposition 2. Let $F: \mathbb{R}^{n} \rightarrow \mathbb{R}^{n}$ be continuous, $\beta \in(0,1), \gamma \geq 0$ and

$$
\|x-F(x)\| \leq \beta\|x\|+\gamma, \quad x \in \mathbb{R}^{n} .
$$

Then $F$ is surjective.

Proposition 2 is a consequence of [2], p. 19, Theorem 3.3.

Proof of Theorem 1. Fix $u_{0} \in \mathbb{R}^{n}$.

For each $k \in \mathbb{N}$ there exists a locally Lipschitz continuous function $f_{k}:[0,1] \times$ $\mathbb{R}^{2 n} \rightarrow \mathbb{R}^{n}$ such that $\left\|f_{k}(t, x, p)-f(t, x, p)\right\| \leq 1 / k,(t, x, p) \in[0,1] \times \mathbb{R}^{2 n}$; see, for example, [1], p. 5. For each $k \in \mathbb{N}$ and $\|x\|+\|p\|>0$ we have

$$
\frac{\left\|f_{k}(\cdot, x, p)-A(\cdot) x-B(\cdot) p\right\| \mid}{\|x\|+\|p\|} \leq \frac{1+\|f(\cdot, x, p)-A(\cdot) x-B(\cdot) p\| \|}{\|x\|+\|p\|} .
$$

Let $0<\varepsilon \leq 1$. We can choose $r>0$ such that for each $k \in \mathbb{N}$,

$$
\left\|f_{k}(t, x, p)-A(t) x-B(t) p\right\| \leq \varepsilon(\|x\|+\|p\|), t \in[0,1],\|x\|+\|p\| \geq r .
$$

We define $g_{k}, h_{k}:[0,1] \times \mathbb{R}^{2 n} \rightarrow \mathbb{R}^{n}$ by

$$
\begin{gathered}
g_{k}(t, x, p)=f_{k}(t, x, p),\|x\|+\|p\|>r ; \quad g_{k}(t, 0,0)=0 ; \\
g_{k}(t, x, p)=\frac{\|x\|+\|p\|}{r} f_{k}\left(t, \frac{r x}{\|x\|+\|p\|}, \frac{r p}{\|x\|+\|p\|}\right), 0<\|x\|+\|p\| \leq r,
\end{gathered}
$$

and $h_{k}=f_{k}-g_{k}$. For each $k \in \mathbb{N}$ the functions $g_{k}, h_{k}$ are continuous,

$$
\left\|g_{k}(t, x, p)-A(t) x-B(t) p\right\| \leq \varepsilon(\|x\|+\|p\|), \quad(t, x, p) \in[0,1] \times \mathbb{R}^{2 n},
$$

and

$$
\left\|h_{k}(t, x, p)\right\| \leq 2\left(\max _{\|\xi\|+\|\eta\| \leq r}\|\| f(\cdot, \xi, \eta)\|\|+1\right)=: K(r),(t, x, p) \in[0,1] \times \mathbb{R}^{2 n} .
$$


We will use the decompositions $f_{k}=g_{k}+h_{k}$ twice. First set $\varepsilon=1$ with corresponding $r=r_{1}$. Consider

$$
v_{k}^{\prime \prime}(t)=f_{k}\left(t, v_{k}(t), v_{k}^{\prime}(t)\right), \quad v_{k}(0)=u_{0}, v_{k}^{\prime}(0)=s .
$$

From the properties of $g_{k}$ and $h_{k}$ we get for $(t, x, p) \in[0,1] \times \mathbb{R}^{2 n}$

$$
\left\|f_{k}(t, x, p)\right\| \leq\left(1+\max _{t \in[0,1]}\|A(t)\|+\max _{t \in[0,1]}\|B(t)\|\right)(\|x\|+\|p\|)+K\left(r_{1}\right) .
$$

Therefore (3) is uniquely solvable on $[0,1]$ (hence we have a continuous shooting function $q_{k}: \mathbb{R}^{n} \rightarrow \mathbb{R}^{n}, q_{k}(s)=v_{k}(1, s)$ for each $\left.k \in \mathbb{N}\right)$, and there are constants $K_{1}, K_{2}>0$ independent of $k$ such that

$$
\left\|v_{k}(t, s)\right\|+\left\|v_{k}^{\prime}(t, s)\right\| \leq K_{1}\|s\|+K_{2}, \quad t \in[0,1], s \in \mathbb{R}^{n} .
$$

Now we choose $\varepsilon \in(0,1)$ (with corresponding $\left.g_{k}, h_{k}, r\right)$ such that $\beta:=\alpha \varepsilon K_{1}<1$, and we set $\gamma:=\alpha \varepsilon K_{2}+\alpha K(r)$.

For each $k \in \mathbb{N}$ consider the solution $w_{k}(t, s)$ of the boundary value problem

$$
w_{k}^{\prime \prime}(t)=A(t) w_{k}(t)+B(t) w_{k}^{\prime}(t), \quad w_{k}(0)=u_{0}, w_{k}(1)=q_{k}(s) .
$$

We have

$$
\begin{gathered}
v_{k}^{\prime \prime}(t, s)-w_{k}^{\prime \prime}(t, s)=A(t)\left(v_{k}(t, s)-w_{k}(t, s)\right)+B(t)\left(v_{k}^{\prime}(t, s)-w_{k}^{\prime}(t, s)\right) \\
+g_{k}\left(t, v_{k}(t, s), v_{k}^{\prime}(t, s)\right)-A(t) v_{k}(t, s)-B(t) v_{k}^{\prime}(t, s)+h_{k}\left(t, v_{k}(t, s), v_{k}^{\prime}(t, s)\right),
\end{gathered}
$$

and

$$
v_{k}(0, s)-w_{k}(0, s)=v_{k}(1, s)-w_{k}(1, s)=0 .
$$

According to Proposition 1

$$
\begin{gathered}
\left\|v_{k}^{\prime}(t, s)-w_{k}^{\prime}(t, s)\right\| \\
\leq \alpha\|\| g_{k}\left(\cdot, v_{k}(\cdot, s), v_{k}^{\prime}(\cdot, s)\right)-A(\cdot) v_{k}(\cdot, s)-B(\cdot) v_{k}^{\prime}(\cdot, s)+h_{k}\left(\cdot, v_{k}(\cdot, s), v_{k}^{\prime}(\cdot, s)\right)\|\| \\
\leq \alpha\left(\varepsilon\left(\|\mid\| v_{k}(\cdot, s)\|\|+\left\|v_{k}^{\prime}(\cdot, s)\right\| \|\right)+K(r)\right) \leq \alpha \varepsilon K_{1}\|s\|+\alpha \varepsilon K_{2}+\alpha K(r), t \in[0,1] .
\end{gathered}
$$

Setting $t=0$ we obtain

$$
\left\|s-w_{k}^{\prime}(0, s)\right\| \leq \beta\|s\|+\gamma, \quad s \in \mathbb{R}^{n} .
$$

According to Proposition 1 the function $w_{k}^{\prime}(0, s), s \in \mathbb{R}^{n}$ is continuous and according to Proposition 2 it is surjective. Since the shooting function of $w^{\prime \prime}=$ $A(t) w+B(t) w^{\prime}$ (with respect to $u_{0}$ ) is bijective we have that $q_{k}$ is surjective, $k \in \mathbb{N}$.

Now let $s_{k}, k \in \mathbb{N}$ be such that the shooting functions $q_{k}$ hit, i.e. $q_{k}\left(s_{k}\right)=u_{1}$, $k \in \mathbb{N}$. Then $u_{k}(t)=v_{k}(t, s)$ solves $u_{k}^{\prime \prime}=f_{k}\left(t, u_{k}(t), u_{k}^{\prime}(t)\right), u_{k}(0)=u_{0}, u_{k}(0)=u_{1}$, and from (4) and (5) we obtain

$$
\left\|u_{k}(t)\right\|+\left\|u_{k}^{\prime}(t)\right\| \leq K_{1}\left\|s_{k}\right\|+K_{2} \leq \frac{K_{1}}{1-\beta}\left(\left\|w_{k}^{\prime}\left(0, s_{k}\right)\right\|+\gamma\right)+K_{2} .
$$

Note that $w_{k}^{\prime}\left(0, s_{k}\right)$ is uniquely determined by $u_{1}$, hence independent of $k$. Therefore, the sequences $\left(u_{k}\right)_{k=1}^{\infty}$ and $\left(u_{k}^{\prime}\right)_{k=1}^{\infty}$ are bounded and equicontinuous. The existence of a solution of problem (1) follows by Ascoli-Arzelà's theorem. 


\section{REFERENCES}

[1] Deimling, K.: Ordinary differential equations in Banach spaces. Lecture Notes in Mathematics, 596, Springer, 1977. MR 57:3546

[2] Deimling, K.: Nonlinear functional analysis. Springer, 1985. MR 86j:47001

[3] Hartman, P.: Ordinary Differential equations. John Wiley \& Sons, 1964. MR 30:1270

[4] Herzog, G., Lemmert, R.: On the structure of the solution set of $u^{\prime \prime}=f(t, u), u(0)=$ $u(1)=0$. Math. Nachr. (to appear).

[5] Lemmert, R.: The shooting method for some nonlinear Sturm-Liouville boundary value problems. J. Appl. Math. Phys. 40 (1989), 769-773. MR 90m:65153

[6] Krasnoselskii, M.A., Perow, A.I., Powolozki, A.I., Sabrejko, P.P.: Vektorfelder in der Ebene. Akademie-Verlag Berlin, 1966. MR 34:1995

Mathematisches Institut I, Universität Karlsruhe, D-76128 Karlsruhe, Germany

E-mail address: Gerd.Herzog@math.uni-karlsruhe.de

E-mail address: Roland.Lemmert@math.uni-karlsruhe.de 